DOI: 10.17117/na.2016.08.01.264

Поступила (Received): 24.08.2016 http://ucom.ru/doc/na.2016.08.01.264.pdf

\title{
Герасимов С.A.
}

\section{Экспериментально о магнитогидродинамике}

неоднородно намагниченного тела

в жидкой и газовой среде

\author{
Gerasimov S.A. \\ Experimentally about magnetohydrodynamics of a non-uniformly \\ magnetized body in fluid and gas medium
}

Воздух, являясь парамагнетиком, должен втягиваться в область максимальной неоднородности магнитного поля. Сила, равная по величине и противоположная по направлению силе, испытываемой парамагнитной средой, действует на находящееся в среде неоднородно намагниченное тело. С другой стороны, на то же тело должно оказывать влияние давление среды, вызванное намагниченностью. Поэтому полная сила, обусловленная магнитодинамическим воздействием и действующая на неоднородно намагниченное находящееся в среде тело должна быть равна нулю. Для газовой среды это действительно так и с высокой точностью подтверждается экспериментально.

Экспериментальные результаты, приведенные для жидких парамагнитной и диамагнитной сред, свидетельствуют о нарушении этого правила. Полная магнитодинамическая сила, обусловленная взаимодействием намагниченного тела с полубесконечной диамагнитной и парамагнитной средой, не равна нулю и зависит от индукции магнитного поля в среде

Ключевые слова: магнитогидродинамика, парамагнетик, диамагнетик, пондеромоторная сила, воздух, давление

\section{Герасимов Сергей Анатольевич}

Кандидат физико-математических наук, доцент Южный федеральный университет

2. Ростов-на-Дону, ул. Зорге, 5
Air as a paramagnetic medium must attract to the place where the magnetic field is non-uniform. $A$ force equal and opposite to that exerted by the paramagnetic substance must act on the nonuniformly magnetized body located in the medium. On the other side the same magnetized body must exert the pressure due to the magnetization of medium. The net force acting on the body must be equal zero. This is true for gas medium and confirmed experimentally with high accuracy. Experimental results obtained for fluid diamagnetic and paramagnetic media demonstrate a violation of such a conclusion. The net force due to the magnetodynamic interaction of the uniformly magnetized body with semi-infinite diamagnetic and paramagnetic medium is equal to zero and depends on the magnetic field induction in the medium

Key words: magnetohydrodynamics, paramagnetic, diamagnetic, ponderomotive force, air, pressure

\author{
Gerasimov Sergey Anatolievich \\ Candidate of Sciences in Physics and Mathematics, \\ Associate Professor \\ Southern federal university \\ Rostov-on-Don, Zorge st., 5
}

Парамагнитная среда, чем, к примеру, является воздух, должна втягиваться в область максимальной неоднородности магнитного поля [1]. В свою 
очередь, на неоднородно намагниченное тело должна действовать сила, равная по величине и противоположная силе, с которой неоднородный магнит действует на воздух. Действие магнитного поля на среду сопровождается возникновением избытка и дефицита давления, поверхностный интеграл от которого по замкнутой поверхности намагниченного тела должен быть в точности равен силе, действующей на помещенный в среду магнит [2]. Обе силы действуют на одно и то же тело, поэтому сумма этих сил в принципе должна быть равна нулю. Это полностью укладывается в рамки закона сохранения импульса: для неравенства полной силы, действующей на тело, задача должна быть не изотропной. С законом сохранения энергии в такой задаче все нормально: для того, чтобы намагнить то или иное тело, нужно затратить энергию. Однако в электродинамике есть экспериментальное свидетельство существования отличной от нуля силы, действующей на систему, помещенную в изотропную среду. Это - так называемая задача о лифтере [3]. Несимметричный заряженный конденсатор, находящийся в газовой среде, испытывает действие отличной от нуля силы, которая играет роль подъемной. Уравнения электродинамики симметричны по отношению к электрическому и магнитному полям. Поэтому, то, что обусловлено воздействием электрического поля на среду, в известных пределах должно быть аналогично действию магнитного поля на вещество.

Первые попытки обнаружить такую парамагнитную силу в воздухе оказались неудачными $[4,5]$. Не удалось даже уверенно заключить, что полная сила, действующая на неоднородно намагниченное тело, находящееся в воздухе, строго равна нулю. Причина, судя по всему, кроется в неудачной постановке эксперимента. Во-первых, намагниченное тело должно быть неподвижно. В противном случае за эффект можно выдать все, что угодно: от влияния окружающей среды до влияния магнитного поля Земли [5]. Во-вторых, погрешности, сопровождающие манипуляции при замене неоднородно намагниченного тела однородным, должны быть сведены к минимуму. Что касается привлечения торсионных (крутильных) колебаний для измерения силы или момента сил [4], то такую методику едва ли можно признать удачной. Дело в том, что коэффициент жесткости подвеса, вообще говоря, зависит от силы натяжения нити, играющей роль подвеса.

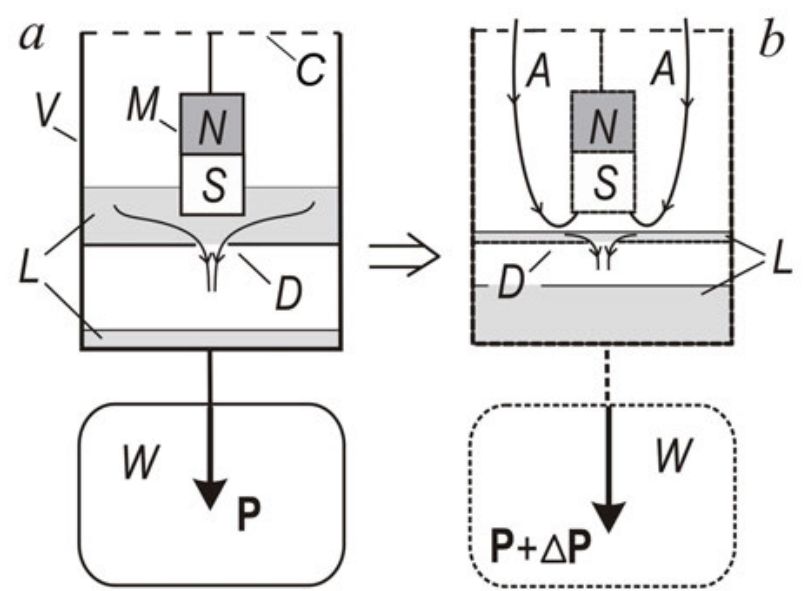

Рис. 1. Эксперимент 1 
Наиболее адекватной и отвечающей постановке задачи является следующая постановка эксперимента (рис. 1). Неоднородно намагниченное тело представляет собой систему из пластмассового стакана $V$ с жидкостью $L$, к верхней крышке $C$ которого прикреплен неодимовый магнит $M$ диаметром 12 мм и высотой 18 мм. Стакан разделен диафрагмой $D$, позволяющей жидкости медленно перетекать из верхней части стакана в нижнюю. Конструкция крышки допускает беспрепятственный доступ воздуха к поверхности жидкости и магнита. Намагниченность магнита (плотность поверхностного молекулярного тока) $\approx$ $10^{6} \mathrm{~A} / \mathrm{м}$. Стакан установлен на чашке прецизионных весов $W$, позволяющих определять массу того или иного тела с точностью не хуже $10^{-5} \mathrm{r}$. С точностью до процессов переноса (испарение, адгезия) и магнитодинамического взаимодействия магнита с окружающей средой система является замкнутой.

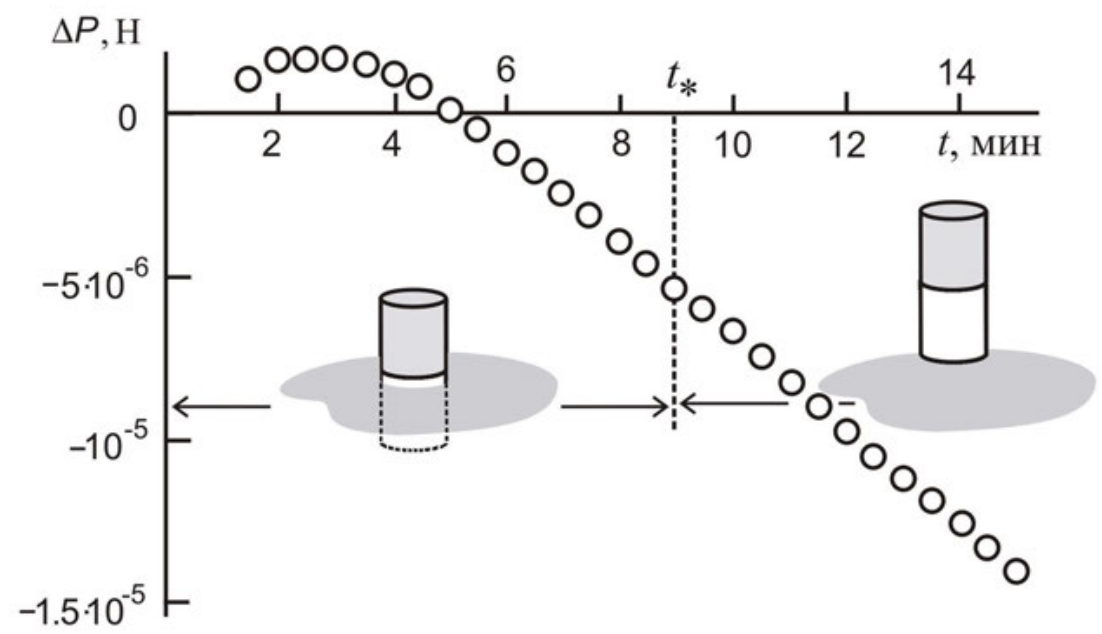

Рис. 2. зависимость веса системы от времени

До тех пор, пока магнит частично погружен в жидкость, изменение веса $\Delta P=P(t)-P(t=0)$ обусловлено только процессами переноса, например, испарением (рис. 1a). Но испарение - процесс медленный, что позволяет его однозначно отличить от магнитодинамического взаимодействия магнита с воздухом. В тот момент времени $t$, когда нижнее основание цилиндрического однородно намагниченного тела $M$ отрывается от поверхности жидкости, вес всей системы должен резко увеличиться (рис. $1 b$ ). Для указанных выше параметров магнита избыток веса должен составить величину, около $5 \cdot 10^{-5} \mathrm{H}$ [6], то есть на полтора порядка больше чувствительности весов. На зависимости изменения веса от времени (рис. 2) это было бы точно заметно. Ничего, заметно отличающегося от изменения веса, обусловленного испарением, не обнаружено. Даже если воздух действительно притягивается к источнику магнитного поля, то его воздействие на магнит, вероятно, полностью компенсируется давлением на магнит, создаваемым воздухом.

Может сложиться впечатление, что приведенные выше результаты представляют собой очередную попытку опровергнуть или подтвердить закон сохранения импульса, а раз так, то под сомнение может быть поставлена сама постановка задачи. 0 конечном результате можно было догадаться заранее. Крыло 
летательного аппарата создает подъемную силу вовсе не потому, что в соответствии с законом Бернулли давление под крылом больше, чем над ним, а потому, что крыло направляет поток воздуха вниз. Такое замечание здесь вполне уместно: для того, чтобы неоднородно намагниченное тело испытало действие “подъемной" силы $-\Delta P$, должен существовать поток среды, направленный в сторону, противоположную подъемной силе. Поэтому здесь обязательно необходимы комментарии или оправдания, если угодно, подтверждающие смысл и целесообразность задачи. Всякая работа, тем более экспериментальная, должна быть чем-то вызвана или обусловлена. Дело в том, что предварительные результаты, вообще говоря ставящие под сомнение то, что сказано выше, хотя и требуют уточнения, но все-таки существуют $[7,8]$.

Считается, что все, что происходит в гидродинамике, с успехом может быть перенесено на аэродинамику [9]. Если это так, то и в жидкости, диамагнитной или парамагнитной, полная сила, действующая на неоднородно намагниченное тело должна быть строго равна нулю. С другой стороны, магнитная восприимчивость даже воды во много раз превышает восприимчивость воздуха. А это - вполне обоснованный повод выяснить особенности магнитодинамического взаимодействия намагниченного тела с жидкостью. Вода, к примеру, в отличие от воздуха, практически не сжимаема. Другое дело, что постановка эксперимента должна быть кардинально изменена, поскольку в первом эксперименте (рис. 1) жидкость уже сыграла свою роль тела, делающего однородно намагниченное тело $(M)$ магнитно-неоднородным $(M+L)$.

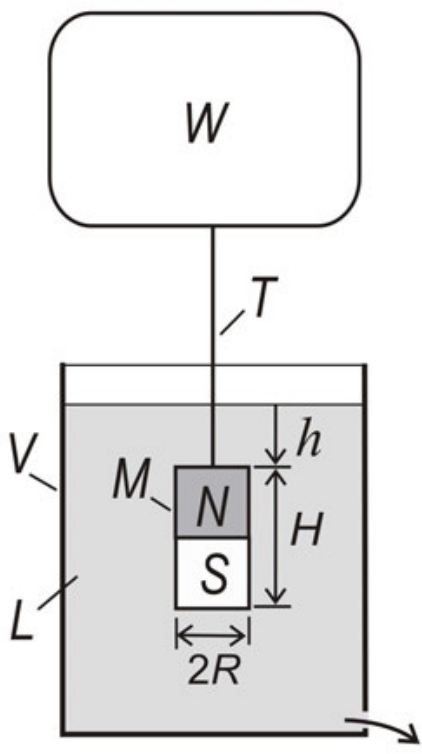

Рис. 3. Эксперимент 2

Теперь магнит $M$ изначально погружен в жидкость $L$ полностью (рис. 3$)$. При больших положительных глубинах погружения магнитодинамическое взаимодействие северного полюса магнита полностью компенсируется взаимодействием южного полюса с той же жидкостью. При вытекании жидкости, кстати говоря, позволяющем определять глубину погружения $h$, слой жидкости над 
магнитом становится меньше, что приводит к ослаблению магнитодинамического воздействия жидкости на магнит. Если жидкость является парамагнитной, вес магнита, измеряемый весами $W$, по мере вытекания жидкости должен уменьшаться. Закон Архимеда, даже в его расширенной форме, позволяющей учитывать процессы переноса [10], здесь не причем: нить $T$, соединяющая магнит с весами, слишком тонка, чтобы вносить более или менее существенный вклад в силу Архимеда.

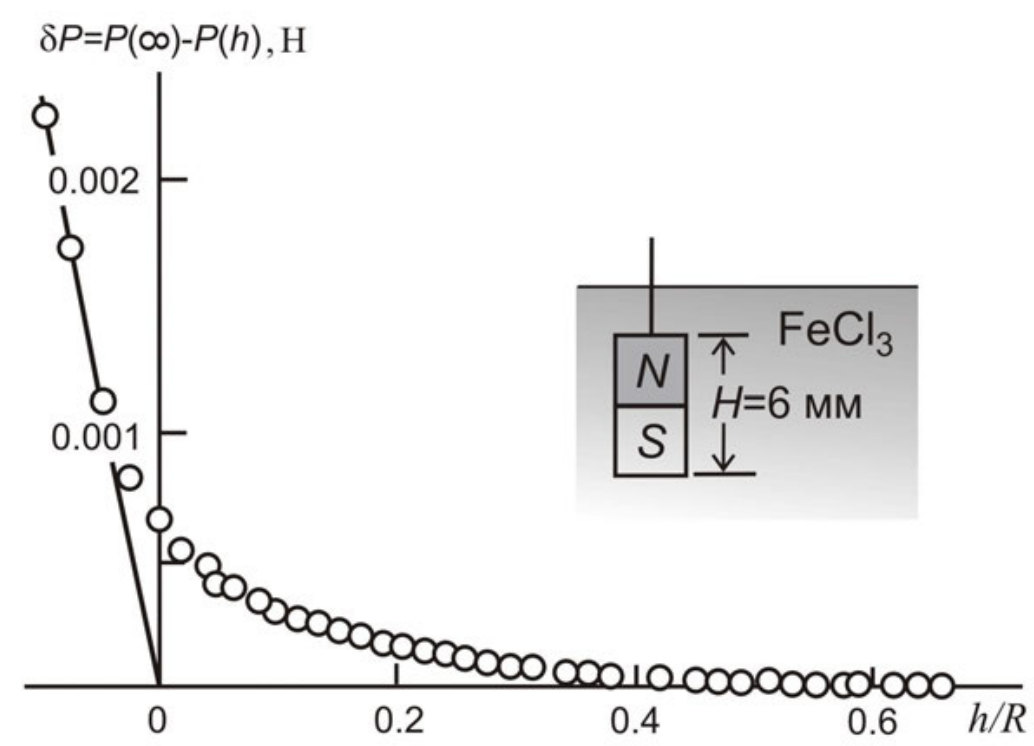

Pис. 4. Вес магнита в 8\% водном растворе хлорида железа. Сплошная линия - сила Архимеда

Достаточно эффективным парамагнетиком является водный раствор хлорного железа. Для его 8\% раствора магнитная восприимчивость составляет $\chi \approx 0,25 \cdot 10^{-3}$, что позволяет зарегистрировать эффект, если он существует, наиболее уверенно. Под изменением веса $\delta P$ в таких измерениях целесообразно понимать вес $P(\infty)$ при большом погружении магнита в хлорид железа III $(h \rightarrow \infty)$ минус вес магнита $P(h)$ при заданной глубине погружения $h$. Это очень удобно, поскольку есть возможность подробно проследить переход к силе Архимеда при отрицательных значениях $h$.

Эффект, то есть взаимодействие даже сравнительно тонкого магнита высотой 6 мм с парамагнитной средой, начинает себя проявлять уже при глубинах погружения, составляющих половину высоты магнита (рис. 4). Объяснить такое изменение веса чем-либо другим, включая особенности закона Архимеда [10], пока не удается. Что касается сравнения настоящих экспериментальных данных с результатами расчета, то здесь лучше сослаться на предварительные оценки $[7,8]$. Не только по порядку величины, не только качественно, но и количественно можно уверено утверждать, что в такой постановке экспериментальной задачи магнит, находящийся в парамагнитной жидкости, испытывает действие существенной силы, уменьшающей его вес. Получается, что при уменьше- 
нии глубины погружения, то есть при уменьшении толщины слоя парамагнитной жидкости, воздействие магнитного поля на среду ослабевает, что влечет к его уменьшению веса, а значит увеличению $\delta P$ (рис. 4 ).

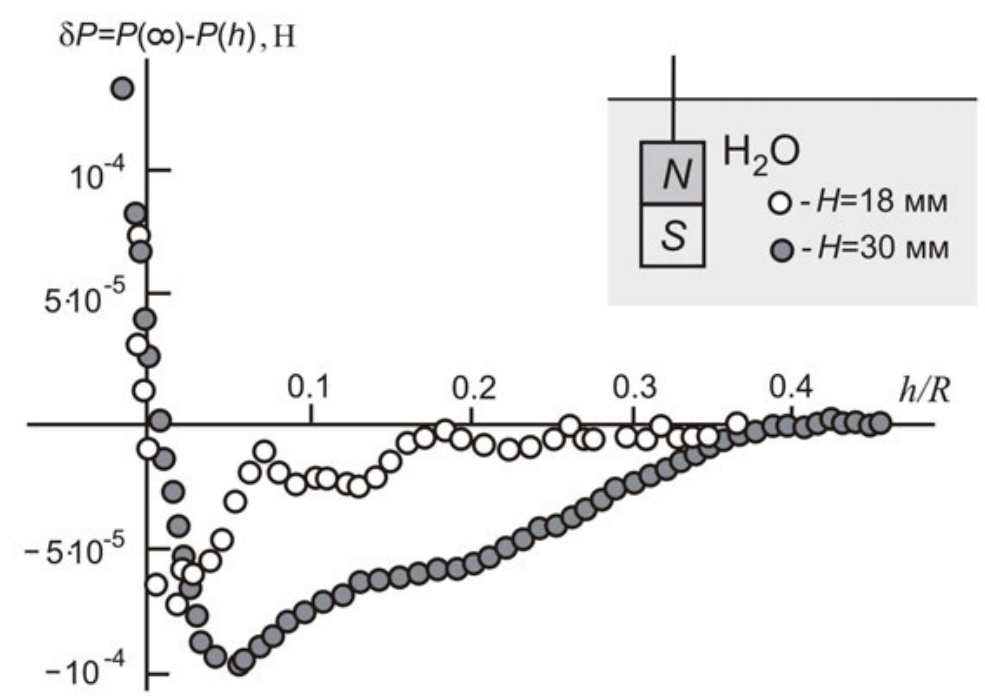

\section{Рис. 5. Зависимость изменения веса от глубины погружения в воду при различных высотах магнита H}

Если все это действительно так, то в случае диамагнитной среды все должно происходить с точностью до наоборот. При этом, правда следует учитывать, что магнитная восприимчивость воды достаточно мала, поэтому эффект на сколько это возможно должен быть усилен, например увеличением магнитного поля с сохранением значения намагниченности. Это позволило не только подтвердить неравенство полной силы, действующее на находящееся в среде намагниченное тело, но и подчеркнуть влияние напряженности или индукции магнитного поля на изменение веса (рис. 5). При уменьшении глубины погружения уменьшается толщина слоя воды над магнитом; сила отталкивания магнита от этого слоя диамагнитной среды уменьшается, а от слоя воды под магнитом - остается прежней; вес тела при заданном значении $h$ увеличивается, а это, в свою очередь, приводит к уменьшению величины $\delta P$.

Самое время попытаться разобраться с происходящим, помня о том, что жидкость в отличие от воздуха практически несжимаема. Несжимаемость жидкости означает не только постоянство плотности жидкости, но и еще одно очень важное свойство. Давление в газе главным образом связано с кинетической энергией молекул, а в жидкости - еще и с потенциальной энергией взаимодействия молекул. Скорость передачи возмущения в несжимаемой жидкости, вообще говоря, бесконечна, то есть любое возмущение должно немедленно передаваться всему объему жидкости [11]. Другими словами, в любой точке несжимаемой жидкости, не подверженной действию внешних сил, давление можно считать постоянным. 


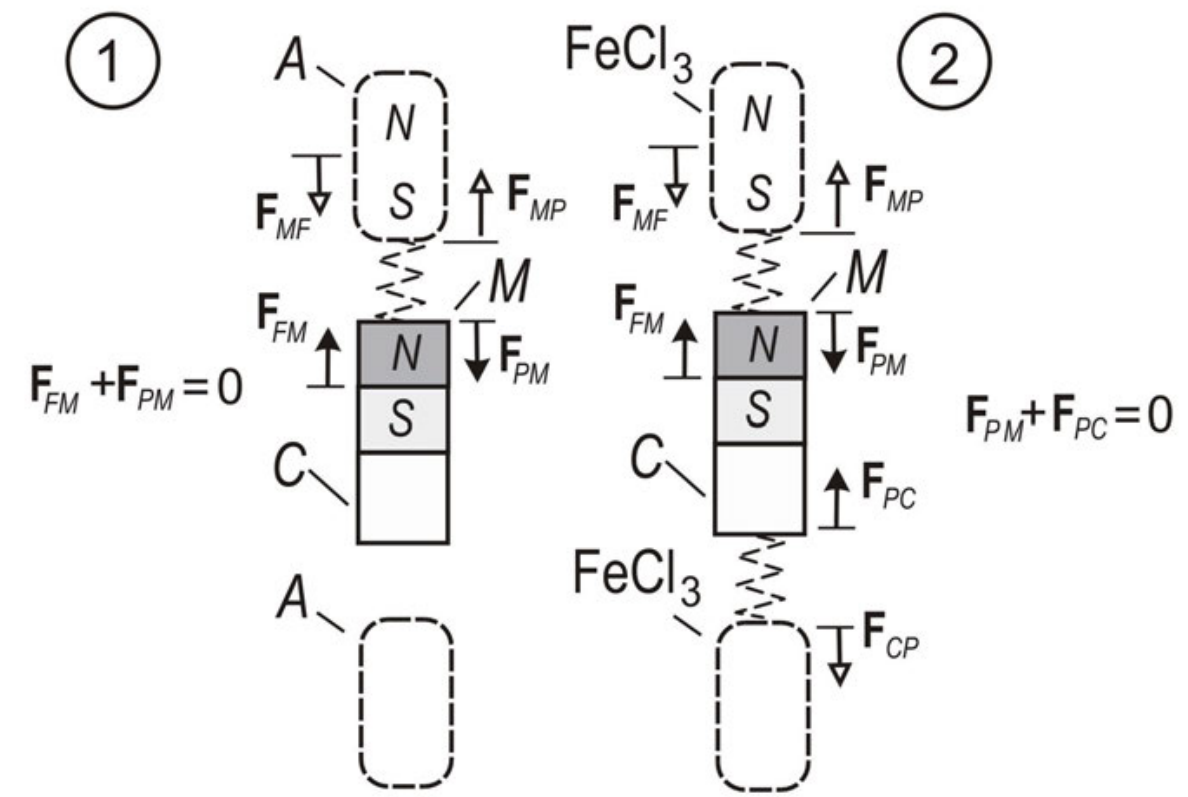

Pис. 6. Модель взаимодействия неоднородно намагниченного тела с газовой средой (1) и несжимаемой жидкостью (2)

В газовой среде неоднородно намагниченное тело (магнит $M$ с присоединенным к нему цилиндром C) притягивается к воздуху с силой $\mathbf{F}_{F M}$, и на этот же полюс действует среда с силой $\mathbf{F}_{P M}$, равной по величине и противоположной по направлению силе $\mathbf{F}_{F M}$ (рис. 6(1)). С другой стороны магнита поле слабо, поэтому неоднородно намагниченное тело ведет себя как однородное; сумма всех внутренних сил, действующих в системе (магнит+газ), равна нулю; полная сила действующая на $M+C$ равна нулю.

В несжимаемой жидкости все должно происходить по-другому. Сила, с которой намагниченная одним полюсом магнита жидкость действует не только на этот полюс, но и на нижнее основание немагнитного цилиндра с силой $\mathbf{F}_{P C}$, равной по величине и противоположной по направлению силе $\mathbf{F}_{P м}$. Поэтому

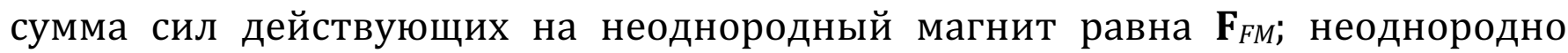
намагниченное тело $M+C$ притягивается к парамагнетику (рис. 6(2)). Как и прежде, сумма всех внутренних сил, действующих в замкнутой системе (магнит+жидкость) равна нулю: закон сохранения импульса, являющийся основным для данной задачи, выполняется. Получается, что при некоторых, пока правда не реальных условиях, магнит может в парамагнитной жидкости даже плавать.

Едва ли следует настаивать на абсолютной справедливости такой модели взаимодействия намагниченного тела с веществом. Дело в том, что при изменении ориентации системы, из вертикального положения в горизонтальное, все сказанное должно иметь место, а эксперимент [4] это не подтверждает. Это означает только одно: работа не закончена, продолжение должно последовать, а упомянутый эксперимент [4] не является чистым. Здесь же подчеркнуто лишь то, что вытекает из настоящих измерений, далеко не последних. 


\section{Список используемых источников:}

1. Макаров В.П., Рухадзе А.А. Сила, действующая на вещество в электромагнитном поле // Успехи физических наук. 2012. T. 179. № 9. С. 995-1001.

2. Ландау Л.Д., Лифшиц Е.М. Гидродинамика. М.: Физматлит, 2006. 736 с.

3. Zhao L., Adamiak K. EHD Gas Flow in Electrostatic Levitation Unit // Journal of Electrostatics. 2006.

V. 64. No 3-4. P. 639-645.

4. Герасимов С.А. Магнит в воздухе и в жидкости: три эксперимента и три нулевых результата // Инженер. 2011. № 3. С. 20-22.

5. Герасимов С.А. Об экспериментальном исследовании обратного парамагнитного эффекта в воздухе // Современные наукоемкие технологии. 2014. № 10. С. 46-49.

6. Герасимов С.А. Намагниченное тело в воздухе: обратный парамагнитный эффект // Инженерная физика. 2011. № 9. С. 15-18.

7. Герасимов С.А. Взаимодействие намагниченного тела с полубесконечной парамагнитной средой // Динамика сложных систем. 2011. Т. 5. № 3. С. 82-84.

8. Gerasimov S.A. Weight of a Magnetized Body in Fluid // Wschodnioeuropejskie Czasopismo Naukovwe. 2016. V. 9. No 4. P. 92-95.

9. Седов Л.И. Методы подобия и размерности в механике. М.: Наука, 1977. 440 с.

10. Герасимов С.А. Сила Архимеда, гидродинамика и скейлинг // Учебная физика. 2015. № 5. С. 35-39. 11. Зарянкин А.Е. Механика несжимаемой и сжимаемой жидкости. М.: Издательский дом МЭИ. 2014. 592 c.

(C) 2016, Герасимов С.A.

Экспериментально о магнитогидродинамике неоднородно намагниченного тела в жидкой и газовой среде
(C) 2016, Gerasimov S.A.

Experimentally about magnetohydrodynamics of a non-uniformly magnetized body in fluid and gas medium 